cultural artefact, but the results of Hignett and Kirkham argue against such a conclusion at least in the case of Venturia.

The Venturia melanoprotein was fractionated on the basis of ethanol solubility and the fraction insoluble in 75 per cent ethanol (N75) was further resolved by gel filtration. The effects of these melanoproteins were studied by injecting them into the petioles of young apple plants and by using non-toxic indicator substances such as glucose labelled with carbon-14. Two important effects were observed. First, there was a marked accumulation of the glucose in the vascular system of the inoculated leaf, whereas the intervenal tissue was unpermeated. The second effect was a strong inhibition of ${ }^{14} \mathrm{C}$ glucose uptake accompanied by an even permeation throughout the leaf. These effects seem to be specific, for a variety of proteins similarly injected did not elicit these responses.

Later experiments have tested whether metabolite transport in plants infected with fungus is disturbed in the same way as plants treated with melanoprotein. Twelve days after fungal inoculation the labelled glucose was accumulating at the sites of presumptive lesions and two days later the distribution of the glucose and the sporulating lesions were identical. Further evidence for the involvement of melanoprotein in lesion development was provided by its co-inoculation with Venturia spores into test plants. A marked stimulation of lesion development occurred when certain fractions of $N 75$ were applied in this way. The melanoprotein fraction responsible for the second effect also has directional properties and prevents movement of metabolites towards the midrib from where they might be transported away from the leaf. Although an accumulation of plant metabolites at lesion sites has been reported in several host-parasite systems, it was not known whether such activity was under the control of host or pathogen. Hignett and Kirkham's observations are therefore an important contribution to the understanding of phytopathogenicity and illustrate a highly sensitive mechanism by which at least one pathogen redirects the metabolism of its host to its own advantage.

\section{Kinds of Ribosomes}

\section{from our Molecular Biology Correspondent}

WITH the isolation of mitochondrial ribosomes by Küntzel and Noll, reported last week in Nature (215, 1340 ; 1967), a clearer pattern of the evolution of the ribosome is beginning to emerge. The mitochondrial ribosomes turn out to have a sedimentation coefficient of $73 \mathrm{~S}$, and to contain RNA components of $20.5 \mathrm{~S}$ and 16.4S. They therefore resemble chloroplast ribosomes $(67 \mathrm{~S}$, with RNA components of $20.8 \mathrm{~S}$ and $15 \cdot 7 \mathrm{~S}$ under the same conditions) and bacterial ribosomes, of which the most familiar example, from $E$. coli, sediment at $70 S$, with RNA of $21 S$ and $16 S$. The possibility that chloroplasts and mitochondria enjoy an independent existence within the cell, and that they originated as some type of symbiotic bacteria, has aroused wide interest.

Apart from size, various other systematic differences between the different classes of ribosomes may, however, be noted. Thus bacterial ribosomes contain about 50 per cent of protein, whereas in plant and animal ribosomes this figure seems always to be near 60 per cent. In terms of base composition of the RNA, animal ribosomes show considerable differences from all others, most noticeably in a consistently higher content of cytosine. It is also relevant that the molecular weight ranges within the above mentioned classes are in fact quite broad-the spread of $66 S$ to $73 S$ in bacterial ribosomes on going from the photosynthetic bacterium $R$. rubrum to $B$. licheniformis, corresponds to a difference of about 25 per cent in molecular weight. (The sedimentation coefficient varies roughly as the square-root of the molecular weight.) Although many of the older data stand in need of revision, and the conditions under which different authors have worked are not always sufficiently alike to permit good comparisons, there is a general tendency, in particular in micro-organisms, of increasing molecular weight with evolutionary status. (Some interesting speculation along these lines is to be found in the article by Küntzel and Noll.)

How these ribosomes differ functionally is a subject of which the surface has so far hardly been scratched, but some interesting experiments on the functioning of heterologous protein synthesizing systems have recently been reported. A revealing study comes from Parisi et al. (J. Mol. Biol., 28, 295; 1967), who have examined poly-U directed cell-free systems with components from two disparate species of bacteria, $E$. coli and $B$. subtilis ( $70 \mathrm{~S}$ ribosomes), and from a micro-organism (yeast), a higher plant (bean seedlings) and an animal tissue (rat liver), all having ribosomes of the $80 \mathrm{~S}$ type. Although the combination of ribosomes of one category with the supernatant enzymes from cells of the other kind gave no significant incorporation, there was substantial incorporation whenever components were mixed within one group. The effect was consistent and striking. To show that the limiting factor was not the failure of synthesis of the aminoacyl-tRNA in the heterologous systems, the experiments were also performed with pre-formed phenylalanyl-tRNA. The source of the latter (whether from the one type of cell or the other) had little effect on the efficiency of incorporation of the phenylalanine. It was also demonstrated that the incompatible systems did not degrade the heterologous aminoacyl- $t \mathrm{RNA}$, neither were any specific inhibitors present in the heterologous supernatants, since these did not interfere when added to functional homologous systems. It appears most probable, therefore, that ribosomes recognize only the relevant protein synthesizing enzymes from sufficiently closely related cells. The similarities between ribosomes within the $70 \mathrm{~S}$ and $80 \mathrm{~S}$ groups therefore go beyond their common physical characteristics, and well into the realm of function.

\section{Peptides without Ribosomes}

\section{from our Cell Biology Correspondent}

Antibiotics like the gramicidins, tyrocidine and licheniformin are short peptide chains of between 10 and about 40 amino-acids synthesized by spore forming bacteria at certain stages in their life cycle. Apart from their antibiotic activity, these peptides are of very great interest because synthesis of them does not involve ribosomes, $m \mathrm{RNA}$ and all the other machinery of protein synthesis. (A group working at the University of Hawaii had claimed that gramicidin is synthesized on ribosomes, but they have recently retracted 